\title{
Quantum Effects in Softly Broken Gauge Theories in Curved Space-Times
}

\author{
I.L. Buchbinder ${ }^{a, b 1}, \quad$ G. de Berredo-Peixoto ${ }^{b}{ }^{2}, \quad$ I.L. Shapiro ${ }^{b} 3$ \\ (a) Department of Theoretical Physics, Tomsk State Pedagogical University \\ 634041, Tomsk, Russia \\ (b) Departamento de Física - ICE, Universidade Federal de Juiz de Fora \\ Juiz de Fora, CEP: 36036-330, MG, Brazil
}

\begin{abstract}
The soft breaking of gauge or other symmetries is the typical Quantum Field Theory phenomenon. In many cases one can apply the Stückelberg procedure, which means introducing some additional field or fields and restore the gauge symmetry. The original softly broken theory corresponds to a particular choice of the gauge fixing condition. In this paper we use this scheme for performing quantum calculations for some softly broken gauge theories in an arbitrary curved space-time. The following examples are treated in details: Proca field, massive QED and massive torsion coupled to fermion. Furthermore we present a qualitative discussions of the discontinuity of quantum effects in the massive spin-2 field theory, paying special attention to the similarity and differences with the massless limit in the spin-1 case.
\end{abstract}

PACS: $04.62 .+\mathrm{v} \quad$ 11.10.Gh 11.15.-q 11.30.-j

Keywords: Curved space-time, Soft symmetry breaking, Renormalization.

\section{Introduction}

Theories with softly broken gauge symmetries emerge frequently in various branches of Quantum Field Theory (QFT). All attempts to consider the soft breaking of the nonabelian gauge symmetry met serious difficulties [1], but anyway they represent an important phase in the development of the modern high energy physics. One more example is supersymmetry which must be (most likely softly) broken in order to address the phenomenological applications and eventually experimental tests [2]. Another interesting application of the soft symmetry breaking is the effective QFT approach to the propagating torsion $[3,4,5]$. The most relevant completely antisymmetric component of torsion can be described by the dual axial vector coupled to fermions through the axial vector current. The presence of the symmetry breaking mass of this axial vector is necessary for the consistency of the effective theory in the low-energy sector. Furthermore one can mention an important problem of discontinuity in the massless limit for the massive spin-2 (sometimes called massive graviton) field [6]. The massless and massive spin-2 particles have different number of degrees of freedom even if the mass is extremely small, hence there is no smooth massless limit, e.g., in the gravitational interaction. The problem of classical discontinuity can be solved if, instead of

\footnotetext{
${ }^{1}$ E-mail address: joseph@tspu.edu.ru

${ }^{2}$ E-mail address: guilherme@fisica.ufjf.br

${ }^{3}$ Also at Tomsk State Pedagogical University, Russia. E-mail address: shapiro@fisica.ufjf.br
} 
the flat background, one takes the curved one [7], which in this case must be dS or AdS space. However, according to the publications [8], the discontinuity persists in the quantum corrections, even in curved space. The last two examples show the importance of evaluating the quantum corrections in the theories with softly broken symmetries, especially in curved space-time.

In the mentioned cases one is interested not only in the classical aspects of the theory, but also in deriving quantum corrections. The subject of the present paper is the calculation of effective action for softly broken gauge theories in curved space-time. In this case a kinetic term and interactions of classical action are gauge invariant while the massive terms are not. As a result the standard methods for evaluating effective action face serious technical difficulties. Our general strategy will be as follows: in each case we shall apply the Stückelberg procedure [9], that is, restore the gauge symmetry by introducing an extra field or a set of fields.

The simplest example is the Proca field model in curved space, which is considered here as a kind of simple pedagogical example, illustrating the method. The restoration of gauge symmetry requires introducing a new scalar field. Then, the original Proca theory corresponds to the special gauge fixing in a new theory, while the quantum calculations are performed in some different gauge, which is most useful from technical viewpoint. Let us notice that the gauge fixing dependence of the effective action should vanish on shell. The situation is especially simple for the one-loop corrections, because in this case the difference between the effective actions calculated in different gauges is proportional to the classical equations of motion. Therefore, when evaluating quantum corrections to the vacuum action (that is the action of external, e.g., gravitational field), the result is gauge fixing independent. If we are dealing with the interacting theory and look also for the renormalization in the matter sector, some additional effort may be requested.

As we shall see in what follows, our approach paves the way for much simpler and more efficient calculation of quantum corrections. The difference is especially explicit for the massive torsion-fermion system which was originally elaborated in [4]. The present method provides an independent verification of our previous result [4] and also enables one to perform the calculations in an arbitrary curved space-time, something that was impossible in the framework used in [4]. The paper is organized as follows. In section 2 we consider quantum calculations for the Proca field in curved space. In section 3 the result is generalized for the massive QED and we also learn some important aspects of dealing with interacting fields. Section 4 is devoted to the massive torsion-fermion system. In section 5 we discuss the one-loop calculations for the massive spin-2 field, especially focusing on the problem of discontinuity of quantum corrections in the massless limit. Finally, in section 6 we draw our conclusions.

\section{Proca theory in curved space}

As a first example, consider the massive Abelian vector fields, which is also called Proca model. The action of the theory in curved space has the form

$$
S_{P}=\int d^{4} x \sqrt{g}\left\{-\frac{1}{4} F_{\mu \nu}^{2}-\frac{1}{2} M^{2} A_{\mu}^{2}\right\} .
$$

Here and throughout the rest of the paper we use the Euclidean signature and condensed notations $F_{\mu \nu}^{2}=F_{\mu \nu} F^{\mu \nu}$ and $A_{\mu}^{2}=A_{\mu} A^{\mu}$. Also, we consistently disregard irrelevant surface terms. The main problem in deriving the quantum corrections here is the softly broken gauge invariance. As a consequence of that, the bilinear form of the action

$$
\hat{H}=H_{\alpha}^{\mu}=\delta_{\alpha}^{\mu} \square-\nabla_{\alpha} \nabla^{\mu}-R_{\alpha}^{\mu}-M^{2} \delta_{\alpha}^{\mu}
$$


is degenerate while the theory is not invariant under the standard gauge transformation. The non-invariance does not permit the use of the usual Faddeev-Popov technique for eliminating the degeneracy. The known way of solving this problem [10] requires introducing an auxiliary operator

$$
\hat{H}^{*}=H_{\mu}^{* \nu}=-\nabla_{\mu} \nabla^{\nu}+M^{2} \delta_{\mu}^{\nu},
$$

which satisfies the following two properties:

$$
\begin{aligned}
H_{\alpha}^{\mu} H_{\mu}^{* \nu} & =M^{2}\left(\delta_{\alpha}^{\nu} \square-R_{\alpha}^{\mu}-M^{2} \delta_{\alpha}^{\mu}\right), \\
\operatorname{Tr} \ln \hat{H}^{*} & =\operatorname{Tr} \ln \left(\square-M^{2}\right) .
\end{aligned}
$$

As a result we arrive at the following relation ${ }^{4}$ :

$$
-\frac{1}{2} \operatorname{Tr} \ln \hat{H}=-\frac{1}{2} \operatorname{Tr} \ln \left(\delta_{\alpha}^{\nu} \square-R_{\alpha}^{\mu}-M^{2} \delta_{\alpha}^{\mu}\right)+\frac{1}{2} \operatorname{Tr} \ln \left(\square-M^{2}\right) .
$$

An obvious advantage of the last formula is that both operators at the r.h.s are not degenerate and admit a simple use of the standard Schwinger-DeWitt technique for the divergences and even the use of a more advanced method for deriving the non-local terms in the second-order in curvature approximation $[12,13]$ Looking at the expression (5) one can observe certain similarity with the massless case. In both cases we meet contributions from minimal vector and scalar operators. Indeed, the second contribution in (5) is analogous to the ghost contribution in the massless case, but there is a factor 1 instead of a factor $1 / 2$ in front of the term $\operatorname{Tr} \ln \square$ in the strictly massless case. As a result of this difference one can observe a discontinuity in the vacuum contribution of massive vector field in the massless limit. The difference between the $M \rightarrow 0$ limit in eq. (5) and the contribution of a massless vector is exactly equal to the contribution of a minimal massless scalar. Which scalar is that?

In order to understand that, let us consider a new action

$$
S_{P}^{\prime}=\int d^{4} x \sqrt{g}\left\{-\frac{1}{4} F_{\mu \nu}^{2}-\frac{1}{2} M^{2}\left(A_{\mu}-\frac{1}{M} \partial_{\mu} \varphi\right)^{2}\right\} .
$$

The remarkable property of this expression is the gauge invariance under the simultaneous transformations $A_{\mu} \rightarrow A_{\mu}^{\prime}=A_{\mu}+\partial_{\mu} \xi$ and $\varphi \rightarrow \varphi^{\prime}=\varphi+\xi M$. Furthermore, in the special gauge $\varphi=0$ we come back to the Proca field action (1). And finally, since both (1) and (6) are free fields actions, the gauge fixing dependence is irrelevant for the quantum correction which depends only on the external metric field.

The last observation means it is not necessary to perform practical calculations in the in the $\varphi=0$ gauge. Instead, one can choose another gauge, e.g., the one which simplifies the Feynman diagrams or the Schwinger-DeWitt technique. Let us use the linear gauge fixing condition $\chi=\nabla_{\mu} A^{\mu}-M \varphi$ for deriving the quantum corrections. Then the sum of the action (6) and the FP gauge fixing term $S_{g f}=-\frac{1}{2} \int d^{4} x \sqrt{g} \chi^{2}$ is cast into the factorized form

$$
S^{\prime}+S_{g f}=\int d^{4} x \sqrt{g}\left\{A^{\alpha}\left(\delta_{\alpha}^{\nu} \square-R_{\alpha}^{\mu}-M^{2} \delta_{\alpha}^{\mu}\right) A_{\nu}+\varphi\left(\square-M^{2}\right) \varphi\right\} .
$$

A simple calculation of the gauge ghost operator gives

$$
\hat{H}_{g h}=\square-M^{2}
$$

\footnotetext{
${ }^{4}$ Indeed the one-loop effective action is given by the formula $\bar{\Gamma}^{(1)}=-\frac{1}{2} \operatorname{Tr} \ln \hat{H}$ (see, e.g., [10, 11]).
} 
and therefore the one-loop effective action is given by

$$
\bar{\Gamma}^{(1)}=-\frac{1}{2} \operatorname{Tr} \ln \left(\delta_{\alpha}^{\nu} \square-R_{\alpha}^{\mu}-M^{2} \delta_{\alpha}^{\mu}\right)-\frac{1}{2} \operatorname{Tr} \ln \left(\square-M^{2}\right)+\operatorname{Tr} \ln \left(\square-M^{2}\right),
$$

that is nothing but (5). One can see that an extra scalar was indeed "hidden" in the massive term of the vector. At this point we conclude that the Stückelberg procedure described above works also in curved space-time and represents a useful alternative to the scheme (3)-(5) for the Proca field in curved space-time [10].

Now we are in a position to discuss the discontinuity in the quantum contributions to the vacuum effective action from the Proca model in the massless limit. For this end we remember the second order in curvatures result for the Proca field derived in [13]

$$
\begin{aligned}
\bar{\Gamma}_{\text {vector }}^{(1)} & =\frac{1}{2(4 \pi)^{2}} \int d^{4} x g^{1 / 2}\left\{\frac{3}{2} M^{4} \cdot\left(\frac{1}{\epsilon}+\frac{3}{2}\right)+\frac{M^{2}}{2} R\left(\frac{1}{\epsilon}+1\right)\right. \\
& \left.+\frac{1}{2} C_{\mu \nu \alpha \beta}\left[\frac{13}{60 \epsilon}+k_{W}^{v}(a)\right] C^{\mu \nu \alpha \beta}+R\left[\frac{1}{72 \epsilon}+k_{R}^{v}(a)\right] R\right\},
\end{aligned}
$$

where

$$
\frac{1}{\epsilon}=\frac{2}{4-n}+\ln \left(\frac{4 \pi \mu^{2}}{M^{2}}\right)-\gamma,
$$

$n$ is the parameter of dimensional regularization and $\gamma$ is the Euler number. The nonlocal finite part of the effective action is characterized by the two formfactors

$$
\begin{aligned}
k_{W}^{v}(a) & =-\frac{91}{450}+\frac{2}{15 a^{2}}-\frac{8 A}{3 a^{2}}+A+\frac{8 A}{5 a^{4}}, \\
k_{R}^{v}(a) & =-\frac{1}{2160}+\frac{A}{48}+\frac{A}{3 a^{4}}+\frac{1}{36 a^{2}}-\frac{A}{18 a^{2}} .
\end{aligned}
$$

Here, $a$ and $A$ are defined according to

$$
A=1-\frac{1}{a} \ln \left|\frac{2+a}{2-a}\right| \quad \text { and } \quad a^{2}=\frac{4 \square}{\square+4 M^{2}} .
$$

The detailed discussion of the massless limit in the formfactor $k_{R}^{v}(a)$ has been given in [13] and also in [14] in relation to the ambiguity of the trace anomaly. Let us now pay attention to another formfactor $k_{W}^{v}(a)$. This term has very special importance because it defines the leading-log quantum contribution to the propagation of the gravitational wave, that is the transverse traceless part of the gravitational perturbation $h_{\mu \nu}=g_{\mu \nu}-g_{\mu \nu}^{(0)}$, where $g_{\mu \nu}^{(0)}$ is the metric of background where the wave is propagating. We can take $g_{\mu \nu}^{(0)}=\eta_{\mu \nu}$ for simplicity. In the limit $M \rightarrow 0$ the expression $k_{W}^{v}(a)$ becomes

$$
k_{W}^{v}(a)=\frac{13}{60}\left(\frac{1}{2-w}-\gamma-\ln \frac{\square}{4 \pi \mu^{2}}\right)-\frac{38}{225} .
$$

The divergent term is cancelled by counterterm and the finite constant terms may be included into renormalization of the $C_{\mu \nu \alpha \beta}^{2}$ term in the classical vacuum action. Finally, the most relevant term is of course the nonlocal one $-\frac{13}{60} \ln \frac{\square}{4 \pi \mu^{2}}$. This term is a physical contribution to the gravitational wave equation for the massless limit of the Proca model. On the other hand, the corresponding term derived for the gauge vector field is just $-\frac{1}{5} \ln \frac{\square}{4 \pi \mu^{2}}$. The difference between the two coefficients is $1 / 60=13 / 60-1 / 5$ is nothing else but the contribution of an extra scalar field which we have discussed before. In the massless limit this field does not disappear and gives contribution to the vacuum effective action. This effect demonstrates the discontinuity in the massless limit for the quantum contributions of the massive (Proca) vector field. 


\section{Massive softly broken QED in curved space}

Our next example is the massive QED in curved space-time. The action has the form

$$
S=S_{P}+S_{F}, \quad S_{F}=\frac{i}{2} \int d^{4} x \sqrt{g}\left\{\bar{\psi} \gamma^{\mu} D_{\mu} \psi-D_{\mu}^{\star} \bar{\psi} \gamma^{\mu} \psi+2 i \bar{\psi} m \psi\right\} .
$$

where $D_{\mu}=\nabla_{\mu}+i q A_{\mu} ; \quad D_{\mu}^{\star}=\nabla_{\mu}-i q A_{\mu}$, the operator $\nabla_{\mu}$ is the covariant derivative acting on Dirac fermion and $S_{P}$ has been defined in eq. (1). The action (14) possesses a softly broken gauge symmetry. The symmetry breaking term is the vector mass, so the situation is very similar to the one in the free Proca field case. There is an important difference, however. The theory (14) includes interaction terms and those terms are gauge invariant. Therefore, in order to restore the gauge symmetry we should not just replace the vector field $A_{\mu}$ by the combination $A_{\mu}-\partial_{\mu} \varphi / M$. At the same time, the procedure described in the previous section works perfectly well, so we now replace the $S_{P}$ in the action (14) by the expression $S^{\prime}$ from (6) and do not modify the term $S_{F}$ in (14). It is easy to see that this procedure restores the symmetry and moreover the consideration of quantum corrections becomes very simple.

In what follows we assume that the quantum corrections are derived within some explicitly covariant and gauge invariant approach, e.g., by using the background field method (see [11] for the introduction). Then in the vacuum (metric-dependent) sector one meets the quantum contributions described in section 2 plus the known contributions from the fermion [13], without additional discontinuity. Furthermore, in the matter sector the situation is rather simple too, at least at the one-loop level. The scalar sector is completely factorized, so the divergences of the theory are the same (except the vacuum ones, described in the previous section) as for the usual massive QED in curved space-time. The only problem which looks nontrivial is the relation between the divergences of the theory with softly broken symmetry (14) and the divergences of the theory $S^{\prime}+S_{F}$ with restored gauge symmetry. In order to address this question we remember that the theory (14) corresponds to the particular gauge fixing condition $\varphi=0$. On the other hand, the loop calculations are performed using the linear gauge fixing. What can be the difference between these two different gauges?

In order to address the last question, let us remember three relevant facts. First, the divergences are local expressions. Second, they are gauge invariant. Third, the difference between the divergent parts of one-loop Effective Actions obtained using two different gauges must be proportional to the classical equations of motion. In our case we denote $\bar{\Gamma}^{(1)}(\chi)$ the effective action corresponding to an arbitrary gauge fixing $\chi$ and $\bar{\Gamma}^{(1)}\left(\chi_{0}\right)$ - to some "minimal" gauge fixing $\chi_{0}$, e.g., the one which is most useful for practical calculations. Then, using the third statement from the list above we arrive at the formula

$$
\bar{\Gamma}^{(1)}\left(\chi_{1}\right)-\bar{\Gamma}^{(1)}\left(\chi_{2}\right)=\int \sqrt{g}\left\{f_{\mu}^{A}(\chi) \frac{\delta S^{\prime}}{\delta A_{\mu}}+f^{\bar{\psi}}(\chi) \frac{\delta S^{\prime}}{\delta \bar{\psi}}+\frac{\delta S^{\prime}}{\delta \psi} f^{\psi}(\chi)+f^{\varphi}(\chi) \frac{\delta S^{\prime}}{\delta \varphi}\right\}
$$

where $f_{\mu}^{A}(\chi), f^{\bar{\psi}}(\chi), f^{\psi}(\chi), f^{\varphi}(\chi)$ are unknown local functions of the background (average) fields. By dimensional reasons and using covariance and Lorentz invariance arguments we arrive at the expressions involving only unknown dimensionless parameters which we can call $p_{1,2,3}$

$$
f_{\mu}^{A}(\chi)=p_{1} A_{\mu}, \quad f^{\bar{\psi}}=p_{2} \bar{\psi}, \quad f^{\psi}(\chi)=p_{2} \psi, \quad f^{\varphi}(\chi)=p_{3} \varphi .
$$

Then, after simple calculations one can obtain

$$
\bar{\Gamma}^{(1)}\left(\chi_{1}\right)-\bar{\Gamma}^{(1)}\left(\chi_{2}\right)=\int \sqrt{g}\left\{-\frac{p_{1}}{2} F_{\alpha \beta}^{2}+p_{1} M^{2} A_{\alpha} A^{\alpha}+\left(p_{3}-p_{1}\right) M A^{\alpha} \partial_{\alpha} \varphi\right.
$$




$$
\left.-p_{3}(\nabla \varphi)^{2}+p_{2} i \bar{\psi}\left(\gamma^{\alpha} \nabla_{\alpha}-i m\right) \psi+\left(p_{1}+p_{2}\right) q \bar{\psi} \gamma^{\alpha} A_{\alpha} \psi\right\}
$$

Now, the requirement of gauge invariance tells us that $p_{1}=p_{3}=0$. Finally, the gauge fixing arbitrariness in the theory under consideration reduces to the usual ambiguity in the renormalization of the fermion wave function. As a result one can safely perform one-loop calculations in the gauge invariant theory with the action $S^{\prime}=S_{P}^{\prime}+S_{F}$ and attribute the result to the theory with the softly broken symmetry (14). The consideration similar to the one presented above can be applied to the much more complicated case of dynamical theory of torsion. We shall discuss this issue in the next section.

\section{Massive torsion coupled to fermion in curved space}

Consider the one-loop renormalization in the coupled torsion-fermion system. This calculation plays an important role for the analysis of the possibility to have a consistent effective quantum field theory of dynamical torsion $[4,5]$.

Torsion $T_{\beta \gamma}^{\alpha}$ is an independent (along with the metric) characteristic of a space-time manifold. It is defined by the relation (see, e.g., $[15,5]$ for introduction)

$$
\Gamma_{\beta \gamma}^{\alpha}-\Gamma_{\gamma \beta}^{\alpha}=T_{\beta \gamma}^{\alpha}
$$

It proves useful to divide torsion into three irreducible components $T_{\mu}, S_{\mu}, q_{\alpha \beta \mu}$ as follows:

$$
T_{\alpha \beta \mu}=\frac{1}{3}\left(T_{\beta} g_{\alpha \mu}-T_{\mu} g_{\alpha \beta}\right)-\frac{1}{6} \varepsilon_{\alpha \beta \mu \nu} S^{\nu}+q_{\alpha \beta \mu} .
$$

Interaction to the Dirac fermion is described by the action $[16,11,5]$

$$
S_{f}=\int d^{4} x \sqrt{g}\left\{i \bar{\psi} \gamma^{\mu}\left(\nabla_{\mu}+i \eta_{1} \gamma^{5} S_{\mu}+i \eta_{2} T_{\mu}\right) \psi+m \bar{\psi} \psi\right\}
$$

where $\eta_{1}, \eta_{2}$ are nonminimal parameters and $\nabla_{\mu}$ is Riemannian covariant derivative (without torsion). The minimal case corresponds to the action

$$
S_{m i n, f}=\frac{i}{2} \int d^{4} x \sqrt{g}\left\{\bar{\psi} \gamma^{\mu} \tilde{\nabla}_{\mu} \psi-\tilde{\nabla}_{\mu}^{\star} \bar{\psi} \gamma^{\mu} \psi-2 i m \bar{\psi} \psi\right\}
$$

where $\tilde{\nabla}_{\mu}$ is the covariant derivative with torsion. Direct calculations show that this action corresponds to the values of the parameters $\eta_{1}=1 / 8, \eta_{2}=0$ in the more general expression (19). The quantum theory meets serious difficulties for a fixed non-zero value of $\eta_{1}$ [16]. Therefore, in what follows we consider $\eta_{1}$ as an arbitrary parameter and keep $\eta_{2}=0$ for simplicity. This is equivalent to requesting that the torsion tensor is completely antisymmetric $T_{\alpha \beta \gamma}=-\frac{1}{6} \varepsilon_{\alpha \beta \gamma \mu} S^{\mu}$.

\subsection{General considerations}

The consistency conditions of an effective quantum fermion-torsion model requires unitarity and renormalizability in the low-energy sector. In this way we arrive at the unique possible form for the action of the theory [3] (see also $[4,5]$ ), where we changed notation from $\eta_{1}$ to $\eta$

$$
S_{t f}=\int d^{4} x \sqrt{g}\left\{-\frac{1}{4} S_{\mu \nu}^{2}-\frac{1}{2} M^{2} S_{\mu}^{2}+i \bar{\psi} \gamma^{\mu}\left(\nabla_{\mu}+i \eta \gamma^{5} S_{\mu}\right) \psi+m \bar{\psi} \psi\right\} .
$$


Here $S_{\mu \nu}=\partial_{\mu} S_{\nu}-\partial_{\nu} S_{\mu}$ and $M$ is the torsion mass, which can not be zero because of the conditions listed above (see $[3,5]$ for more details).

The kinetic and interaction terms of the theory (21) possesses the gauge symmetry

$$
\psi^{\prime}=\exp \left(i \eta \gamma^{5} \beta\right) \psi, \quad \bar{\psi}^{\prime}=\bar{\psi} \exp \left(i \eta \gamma^{5} \beta\right), \quad S_{\mu}^{\prime}=S_{\mu}-\partial_{\mu} \beta, \quad \text { where } \beta=\beta(x) .
$$

Both massive terms are not invariant under these transformations and hence the symmetry is softly broken. In order to learn the implications of this fact we need to evaluate one-loop and two-loop quantum corrections [4]. The main practical difficulty comes from the one-loop divergences calculus. The method of derivation developed in [4] has been based on the Boulware parametrization for a massive vector [17]. This approach is efficient but rather complicated. In particular, one has to develop a special kind of Schwinger-DeWitt expansion in the transverse vector space and work out many universal traces [10]. Technically, this is one of the most complicated one-loop calculations done so far. At the same time the physical output looks remarkable, in particular one can rule out the possibility of an independent light (that is potentially observable) torsion field using the quantum field theory arguments. Therefore, it would be nice to have an independent verification for the result of the mentioned 1-loop calculations. The Stückelberg procedure [9] opens the door for solving this problem.

Following the approach discussed in the previous sections, we introduce a new scalar field $\varphi$ and restore the gauge symmetry in a following way:

$$
\begin{aligned}
S_{t f}^{\prime} & =\int d^{4} x \sqrt{g}\left\{-\frac{1}{4} S_{\mu \nu}^{2}+\frac{1}{2} M^{2}\left(S_{\mu}-\frac{\partial_{\mu} \varphi}{M}\right)^{2}\right. \\
& \left.+i \bar{\psi} \gamma^{\mu}\left(\nabla_{\mu}+i \eta_{1} \gamma^{5} S_{\mu}\right) \psi+m \bar{\psi} \exp \left(\frac{2 i \eta \gamma^{5} \varphi}{M}\right) \psi\right\},
\end{aligned}
$$

The symmetry transformations (22) must be supplemented by $\varphi \rightarrow \varphi^{\prime}=\varphi-M \beta$. The original theory (21) is restored when we use the gauge fixing condition $\varphi=0$. However, the practical calculations can be performed in some useful linear gauge where the scalar field persists. In this case we meet the new exponential coupling with the negative dimensional coupling. This is a very remarkable occurrence, because it provides a qualitative explanation for the result obtained in [4]. Because of the exponential coupling the theory (23) is not renormalizable and therefore it is not a surprise that we meet the counterterms with the form which is different from the ones in the classical action. In particular, one can easily construct the divergent diagrams which produce the $\frac{1}{M^{2}}(\bar{\psi} \psi)^{2}$-type divergence. Indeed, we really met these divergences in [4] after very complicated calculations. Now we can understand them as manifestation of the loop made from an extra scalar degree of freedom, which was hidden in the original formulation of the theory and became explicit after we used the Stückelberg procedure.

Indeed, the eq. (23) is an essential generalization of the usual Stückelberg procedure [9] for the case of complicated theory. In the next subsection we demonstrate how this new transformation may be applied for quantum calculations.

Before starting the one-loop calculation, let us briefly discuss the gauge fixing dependence in the theory (23). The expression for the difference between two effective actions corresponding to the different gauge fixing conditions is very similar to the one for the massive QED (17)

$$
\begin{array}{r}
\bar{\Gamma}^{(1)}\left(\chi_{1}\right)-\bar{\Gamma}^{(1)}\left(\chi_{2}\right)=\int \sqrt{g}\left\{-\frac{p_{1}}{2} S_{\alpha \beta}^{2}+p_{1} M^{2} S_{\alpha} S^{\alpha}+\left(p_{3}-p_{1}\right) M S^{\alpha} \partial_{\alpha} \varphi-p_{3}(\nabla \varphi)^{2}\right. \\
\left.+p_{2} i \bar{\psi}\left(\gamma^{\alpha} \nabla_{\alpha}-i m\right) \psi+\left(p_{1}+p_{2}\right) q \bar{\psi} \gamma^{\alpha} S_{\alpha} \psi+\frac{2 i m}{M} p_{3} \varphi \bar{\psi} \exp \left(\frac{2 \eta \gamma^{5} \varphi}{M}\right) \psi\right\} .
\end{array}
$$


As in the previous case, the gauge invariance of the divergences requires $p_{1}=p_{3}=0$ and does not impose restrictions on the parameter $p_{2}$. The gauge fixing dependence is reducing to the standard ambiguity in the renormalization of the fermion wave function.

\subsection{One-loop calculation}

In this subsection we shall calculate the one-loop divergences in the theory (23), using the background field method and Schwinger-DeWitt technique of extracting divergences of the Effective Action. After that we shall fix the gauge $\varphi=0$ for the background fields and compare the result with the one obtained in [4] using much more complicated approach. The application of the Stückelberg procedure leads to serious improvement in the calculational procedure. In particular, here we will be able to obtain the divergences in curved space-time and hence our results will be more general than the ones of [4].

The calculation will be performed using background field method and standard (different from [4]) Schwinger-DeWitt technique (see, e.g., [11] for the introduction). The first step is the splitting of the fields into background and quantum ones

$$
S_{\mu} \rightarrow S_{\mu}+s_{\mu}, \quad \psi \rightarrow \psi+\chi, \quad \bar{\psi} \rightarrow \bar{\psi}+\bar{\chi}, \quad \varphi \rightarrow \varphi+\theta
$$

where we kept classical notations for the background fields. The most simple "minimal" gaugefixing term has the form

$$
\mathcal{L}_{\mathrm{GF}}=-\frac{1}{2} \sqrt{g}\left(\nabla_{\mu} s^{\mu}+M \theta\right)^{2} .
$$

The bilinear Lagrangian in quantum fields, including the gauge-fixing term, is given by

$$
L_{t}^{(2)}=\frac{1}{2}\left(\begin{array}{llll}
s_{\mu} & \bar{\chi} & \theta
\end{array}\right)(\hat{\mathcal{H}})\left(\begin{array}{c}
s^{\nu} \\
\chi \\
\theta
\end{array}\right),
$$

where

$$
\hat{\mathcal{H}}=\left(\begin{array}{ccc}
\delta_{\nu}^{\mu} \square-R_{\nu}^{\mu}+M^{2} \delta_{\nu}^{\mu} & 2 \eta \bar{\psi} \gamma^{5} \gamma^{\mu} & 0 \\
2 \eta \gamma^{5} \gamma_{\nu} \psi & 2 i \gamma^{\mu} \nabla_{\mu}+2 \eta \gamma^{5} \gamma^{\mu} S_{\mu} & \frac{4 i \eta m}{M} \gamma^{5} \mathrm{e}^{2 i \eta \varphi \gamma^{5} / M} \psi \\
& +2 m \mathrm{e}^{2 i \eta \varphi \gamma^{5} / M} & \\
0 & \frac{4 i \eta m}{M} \bar{\psi} \gamma^{5} \mathrm{e}^{2 i \eta \varphi \gamma^{5} / M} & -\frac{4 \eta^{2} m}{M^{2}} \bar{\psi} \mathrm{e}^{2 i \eta \varphi \gamma^{5} / M} \psi
\end{array}\right) .
$$

Multiplying $\hat{\mathcal{H}}$ by a matrix $\hat{K}$

$$
\hat{K}=\left(\begin{array}{ccc}
\delta_{\alpha}^{\nu} & 0 & 0 \\
0 & -\frac{1}{2} i \gamma^{\rho} \nabla_{\rho} & 0 \\
0 & 0 & -1
\end{array}\right),
$$

we obtain the operator

$$
\hat{\mathcal{H}} \hat{K}=\hat{1} \square+2 \hat{h}^{\rho} \nabla_{\rho}+\hat{\Pi},
$$


where

$$
\hat{1}=\left(\begin{array}{ccc}
\delta_{\nu}^{\mu} & 0 & 0 \\
0 & \hat{1} & 0 \\
0 & 0 & 1
\end{array}\right), \quad \hat{h}^{\rho}=\left(\begin{array}{ccc}
0 & -\frac{i}{2} \eta \bar{\psi} \gamma^{5} \gamma^{\mu} \gamma^{\rho} & 0 \\
0 & -\frac{i}{2} m \mathrm{e}^{2 i \eta \varphi \gamma^{5} / M} \gamma^{\rho}-\frac{i}{2} \eta \gamma^{5} \gamma^{\alpha} \gamma^{\rho} S_{\alpha} & 0 \\
0 & \frac{\eta m}{M} \bar{\psi} \gamma^{5} \mathrm{e}^{2 i \eta \varphi \gamma^{5} / M} \gamma^{\rho} & 0
\end{array}\right)
$$

and

$$
\hat{\Pi}=\left(\begin{array}{ccc}
-R_{\nu}^{\mu}+M^{2} \delta_{\nu}^{\mu} & 0 & 0 \\
2 \eta \gamma^{5} \gamma_{\nu} \psi & -\frac{\hat{1}}{4} R & -\frac{4 i \eta m}{M} \gamma^{5} \mathrm{e}^{2 i \eta \varphi \gamma^{5} / M} \psi \\
0 & 0 & M^{2}+\frac{4 \eta^{2} m}{M^{2}} \bar{\psi} \mathrm{e}^{2 i \eta \varphi \gamma^{5} / M} \psi
\end{array}\right)
$$

The divergent part of $s \operatorname{Tr} \ln \hat{\mathcal{H}}$ (here $s \operatorname{Tr}$ means functional supertrace, including covariant integration over the spacetime variables, usual trace over spinor indices and taking into account the Grassmann parity of the fields) can be achieved just by calculating $\sin \ln (\hat{\mathcal{H}} \hat{K})$ and then subtracting the $\operatorname{sTr} \ln \hat{K}$

$$
-\frac{1}{2} \mathrm{~s} \operatorname{Tr} \ln \hat{\mathcal{H}}=-\frac{1}{2} \mathrm{~s} \operatorname{Tr} \ln (\hat{\mathcal{H}} \hat{K})+\frac{1}{2} \mathrm{~s} \operatorname{Tr} \ln \hat{K},
$$

where $\sin \ln \hat{K}$ produces only metric-dependent vacuum contributions to the Effective Action. They are well known and in fact irrelevant for our purposes. In what follows we will not consider these terms. As far as the integration over the Faddeev-Popov (FP) ghosts also gives only vacuum (that is depending exclusively on the metric) contributions, one can find the relevant one-loop divergences in the form

$$
\Gamma_{\text {div }}^{(1)}=\left.\frac{i}{2} \mathrm{~s} \operatorname{Tr} \ln (\hat{\mathcal{H}} \hat{K})\right|_{\text {div }} .
$$

The above expression can be calculated by using the standard Schwinger-DeWitt algorithm

$$
-\left.\frac{1}{2} \mathrm{~s} \operatorname{Tr} \ln (\hat{\mathcal{H}} \hat{K})\right|_{\text {div }}=-\frac{\mu^{n-4}}{(4 \pi)^{2}(n-4)} \mathrm{s} \operatorname{Tr}\left\{\frac{1}{2} \hat{P} \hat{P}+\frac{1}{12} \hat{\mathcal{R}}_{\alpha \beta} \hat{\mathcal{R}}^{\alpha \beta}\right\}
$$

where the vacuum terms were omitted and the matrices $\hat{P}$ and $\hat{\mathcal{R}}_{\alpha \beta}$ are given by

$$
\begin{aligned}
\hat{P} & =\hat{\Pi}+\frac{\hat{1}}{6} R-\nabla_{\rho} \hat{h}^{\rho}-\hat{h}_{\rho} \hat{h}^{\rho}, \\
\hat{\mathcal{R}}_{\alpha \beta} & =\left[\nabla_{\beta}, \nabla_{\alpha}\right] \hat{1}+\nabla_{\beta} \hat{h}_{\alpha}-\nabla_{\alpha} \hat{h}_{\beta}+\hat{h}_{\beta} \hat{h}_{\alpha}-\hat{h}_{\alpha} \hat{h}_{\beta} .
\end{aligned}
$$

The straightforward calculation using this formula gives us, after certain algebra, the following result for the relevant terms of the Effective Action:

$$
\begin{aligned}
\Gamma_{\text {div }}^{(1)} & =-\frac{\mu^{n-4}}{(4 \pi)^{2}(n-4)} \int d^{n} x \sqrt{g}\left\{4 \eta^{2} m^{2} S^{\mu}\left(S_{\mu}-\frac{1}{M} \nabla_{\mu} \varphi\right)-\frac{1}{3} \eta^{2} S_{\mu \nu}^{2}+2 i \eta^{2} \bar{\psi} \gamma^{\rho} \mathcal{D}_{\rho} \psi\right. \\
& +\frac{4 i \eta^{2} m^{2}}{M^{2}} \bar{\psi} \gamma^{\rho} \mathcal{D}_{\rho}^{*} \psi+8 \eta^{2} m\left(\frac{m^{2}}{M^{2}}-\frac{1}{2}\right) \bar{\psi} \exp \left(\frac{2 i \eta \varphi \gamma^{5}}{M}\right) \psi \\
& \left.-\frac{8 \eta^{3} m^{2}}{M^{3}} \bar{\psi} \gamma^{\mu} \gamma^{5}\left(\nabla_{\mu} \varphi\right) \psi+\frac{2 \eta^{2} m}{3 M^{2}} \bar{\psi} \mathrm{e}^{2 i \eta \varphi \gamma^{5} / M} R \psi+\frac{8 \eta^{4} m^{2}}{M^{4}}\left(\bar{\psi} \mathrm{e}^{2 i \eta \varphi \gamma^{5} / M} \psi\right)^{2}\right\},
\end{aligned}
$$

where $\mathcal{D}_{\rho}=\nabla_{\rho}+i \eta \gamma^{5} S_{\rho}$ and $\mathcal{D}_{\rho}^{*}=\nabla_{\rho}-i \eta \gamma^{5} S_{\rho}$. Let us notice that this expression represents the divergences of the theory (23), involving axial vector, fermion and scalar, with exponential 
interaction between the last two fields. This result has its own independent merit, especially because the interaction is non-polynomial and the above expression is a sum of an infinite number of Feynman diagrams.

In order to obtain the one-loop divergences for the original theory $(21)$, one has to put $\varphi=0$. Then the above result reduces to

$$
\begin{aligned}
\Gamma_{\mathrm{div}}^{(1)} & =-\frac{\mu^{n-4}}{(4 \pi)^{2}(n-4)} \int d^{n} x \sqrt{g}\left\{4 \eta^{2} m^{2} S^{\mu} S_{\mu}-\frac{1}{3} \eta^{2} S_{\mu \nu}^{2}+4 i \eta^{2} \frac{m^{2}}{M^{2}} \bar{\psi} \gamma^{\mu} \mathcal{D}_{\mu}^{*} \psi+\right. \\
& \left.+2 i \eta^{2} \bar{\psi} \gamma^{\mu} \mathcal{D}_{\mu} \psi+\left(\frac{8 \eta^{2} m^{3}}{M^{2}}-4 \eta^{2} m\right) \bar{\psi} \psi+\frac{2 \eta^{2} m}{3 M^{2}} \bar{\psi} R \psi+\frac{8 \eta^{4} m^{2}}{M^{4}}(\bar{\psi} \psi)^{2}\right\}
\end{aligned}
$$

It is easy to see that the difference with the one-loop result derived by different method in [4] consists in the following two kind of terms:

1) Term proportional to the scalar curvature could not be obtained in [4], because the calculation has been performed in flat space-time.

2) For the flat background, the difference between the two results

$$
\Gamma\{\text { Eq. }(37)\}-\Gamma\{\text { Ref. [4] }\} \quad \propto \quad 2 \eta^{2} \bar{\psi}\left(i \gamma^{\mu} \mathcal{D}_{\mu} \psi+m\right) \psi
$$

is proportional to the classical equations of motion for the fields and hence, according to the considerations presented in the previous section, this difference is due to the distinct parametrizations of the quantum fields in two cases. All in all, the expression (37) represents a perfect fit to the result of the much more involved and cumbersome calculation of [4].

\section{Qualitative discussion of a discontinuity phenomena in massive spin-1 and spin-2 field theories.}

Free massive higher spin field models (see, e.g., [18, 19, 20, 21, 22, 23, 24]) are typical examples of the softly broken gauge theories, where the gauge symmetry is broken by the non-zero mass of the field. The evaluation of effective action in these theories can be carried out with the help of Stückelberg procedure. This procedure has been used for the effective action in the massive spin-1 field model in curved space in Section 2 and in the massive spin-2 field model on AdS space in [8]. We do not intend to perform practical calculations for higher spin fields in the present paper. Instead we shall address one important general aspect of the quantum corrections produced by higher spin fields on curved background. For this end we perform a comparison of the discontinuity phenomena for the massive theories with spin-1 and spin-2.

In the massless limit, in flat space-time, the massive spin-2 field manifests a discontinuity [6] due to the different number of degrees of freedom in the massive and massless cases. The situation may be quite different in the curved space. In particular, it has been shown that the classical discontinuity does not occur in the Anti-de Sitter [20] and in the de Sitter [7] space. At the same time, according to [8] the discontinuity persists in the vacuum quantum corrections generated by the massive spin-2 field.

The calculations performed in [8] are quite similar to the ones we have presented in section 2 for the massive vector field. The Stückelberg procedure requires the introduction of vector and scalar auxiliary fields, which are called to restore the symmetry which is softly broken by massive fields. In the massless limit $m^{2} \rightarrow 0$ the loops of the auxiliary fields do not disappear and 
moreover their number is not the same as the number of the Faddeev-Popov ghosts in the massless diffeomorphism invariant case.

As a result the divergent parts of the vacuum effective actions are indeed different for the massless spin-2 field and for the massless limit of the massive spin- 2 field. At the first sight, the relation between quantum corrections for $m \rightarrow 0$ and $m=0$ cases in the spin-2 theory looks similar to the one in the vector case.

One can notice that there is also a significant difference between the discontinuity of the quantum corrections in the massive spin-1 and spin-2 cases. Let us look again at the expression for the $m \rightarrow 0$ limit of the quantum contribution for the massive spin-1 field (13). As it was already discussed in section 2 , we can distinguish two different parts in this expression. The first is local, it includes the divergent expression. One has to remember that this part has no direct physical sense, for it must be modified by adding a local counterterm in the vacuum sector [11]. After that, the overall coefficient of the local term should be fixed by the renormalization condition. Then, the difference between the theories with different coefficients of the local terms disappears. Of course, the last statement is not really correct in the vector case, where we also meet a very important non-local term, with the coefficient which is equal to the one of the local divergent term. This equality is important, in particular it provides the possibility to derive the renormalization group $\beta$-functions in the $\overline{\mathrm{MS}}$ renormalization scheme.

However, a Lagrangian construction for the free massive spin-2 field model is known only for the spaces of constant curvature (see, e.g., [19, 20, 21, 22, 23, 24]). In such spaces one can not construct the non-local insertions similar to the ones we met in eq. (13). The physical sense of the renormalization group in the vacuum sector becomes, in this case, rather obscure. As a result, all the existing difference between the massless theory and the massless limit of the massive theory can be formally eliminated by the renormalization of vacuum effective action. In spite of the difference between the divergent contributions to vacuum effective action in the two cases $(m=0$ vs $m \rightarrow 0$ ) can be treated as manifestation of discontinuity, it does not automatically imply that there is a discontinuity in the quantum corrections to physically observable quantities. In order to clarify this problem one has to achieve the physical interpretation of the available difference between quantum corrections to renormalized quantities for the cases of zero and non-zero masses in the spin-2 filed theories.

\section{Conclusions}

We have demonstrated the advantages of using the Stückelberg procedure for the purposes of quantum calculations in curved space-time. In the case of massive Abelian vector we have calculated, in a new alternative manner, the one-loop correction to the graviton propagator and investigated the discontinuity of this correction in the massless limit. Furthermore, we applied the same procedure to the two different models of interacting spin-1 and fermion field. In the case of massive QED the one-loop calculation is very simple and we just arrive at the result which can be, in principle, obtained by other methods.

In the case of axial vector (antisymmetric torsion) coupled to fermion we arrived at the new method of calculation which is much better than the previously developed one [4] in many respects. In particular, the calculations become much simpler and they could be completed even in an arbitrary curved background. It is remarkable that in the flat space limit we have confirmed the previous result, obtained by in a much more cumbersome way. The new approach based on the 
Stückelberg procedure for the axial vector, made the statement about the non-renormalizability of the theory much more explicit and, once again, demonstrated an essential similarity with the massive Yang-Mills case [1] (see also recent works [25]).

Finally, we presented a qualitative discussion of a very interesting problem of discontinuity of the massless limit for the massive spin-2 contributions to the renormalization of the vacuum energy. The practical derivation of the one-loop contributions of the spin-2 fields has been performed in [8] by using the Stückelberg procedure. We have argued, using comparison with the spin-1 case that the interpretation of this quantum discontinuity is not obvious at the present state of knowledge about the massive higher spin fields on curved background. At the same time, the calculations of effective action using the Stückelberg procedure can be performed for the massive fields of an arbitrary spins on AdS space. The expected result is qualitatively similar with ones for the $s=2$ case, however the algebraic structure of the relevant operators may be a bit more involved. Of course, a problem of effective action for massive arbitrary higher spin theories on AdS space is interesting and important itself. We plan to report about the practical calculations in the massive $s=3$ and maybe other cases in the near future.

Acknowledgments. I.L.B is grateful to FAPEMIG (Minas Gerais, Brazil) for the finance support of his visit to the Physics Department of UFJF. The work of I.L.B has been partially supported by the grant for LRSS, project No 4489.2006.2, joint RFBR-DFG grant, project No 0602-04012, DFG grant, project No 436 RUS/13/669/0-3 and INTAS grant, project INTAS -05-7928. G.B.P. and I.Sh acknowledge support of CNPq (Brazil), FAPEMIG, FAPES and ICTP.

\section{References}

[1] T. Kunimasa and T. Goto, Prog. Theor. Phys. 37 (1967) 452;

M.J.G. Veltman, Nucl. Phys. B7 (1968) 637;

J.Reiff and M.Veltman, Nucl. Phys. B13 (1969) 545;

A. Salam and J.A. Strathdee, Phys. Rev. D2 (1970) 2869;

A.A. Slavnov and L.D. Faddeev, Theor. Math. Fiz. (in Russian) 3 (1970) 18, English translation: Theor. Math. Phys. 3 (1970) 312;

A.I. Vainshtein and I.B. Khriplovich, Yad. Fiz. (Sov. J. Nucl. Phys.) 13 (1971) 198;

[2] S. Dimopoulos and H. Georgi, Nucl. Phys. B193 (1981) 150; see also

M. Drees, R.M. Godbole and P. Roy, Theory and Phenomenology of SPARTICLES: An account of four-dimensional $N=1$ Supersymmetry in High Energy Physics, (World Scientific, 2004).

[3] A.S. Belyaev and I.L. Shapiro, Phys. Lett. 425B (1998) 246; Nucl. Phys. B543 (1999) 20.

[4] G. de Berredo-Peixoto, J.A. Helayel-Neto and I. L. Shapiro, JHEP 02 (2000) 003.

[5] I.L. Shapiro, Phys. Repts. 357 (2002) 113.

[6] H. van Dam, M.J.G. Veltman, Nucl. Phys. B22 (1970) 397;

V.I. Zakharov, JETP Lett. 12 (1970) 312. 
[7] I.I. Kogan, S. Mouslopoulos, A. Papazoglou, Phys. Lett. B503 (2001) 173;

M. Porrati, Phys. Lett. B498 (2001) 92.

[8] F.A. Dilkes, M.J. Duff, J.K. Liu and H. Sati, Phys. Rev. Lett. 87 (2001) 041301;

M.J. Duff, J.T. Liu and H. Sati, Phys. Lett. B516 (2001) 156.

[9] E.C.G. Stückelberg, Helv. Phys. Acta. 30 (1957) 209.

[10] A.O. Barvinsky and G.A. Vilkovisky, Phys. Rep. 119 (1985) 1.

[11] I.L. Buchbinder, S.D. Odintsov and I.L. Shapiro, Effective Action in Quantum Gravity (IOP Publishing, Bristol, 1992).

[12] A.O. Barvinsky and G.A. Vilkovisky, Nucl. Phys. 333B (1990) 471;

I. G. Avramidi, Yad. Fiz. (Sov. Journ. Nucl. Phys.) 49 (1989) 1185.

[13] E.V. Gorbar and I.L. Shapiro, 06 (2003) 006.

[14] M. Asorey, E.V. Gorbar and I.L. Shapiro, Class. Quant. Grav. 21 (2003) 163.

[15] F.W. Hehl, Gen. Relat. Grav. 4 (1973) 333; 5 (1974) 491;

F.W. Hehl, P. Heide, G.D. Kerlick and J.M. Nester, Rev. Mod. Phys. 48 (1976) 393.

[16] I.L. Buchbinder and I.L. Shapiro, Phys. Lett. B151 (1985) 263.

[17] D. Boulware, Ann. Phys. (NY) 56 (1970) 140-171.

[18] L.P.S. Singh and C.R. Hagen, Phys. Rev. D9 (1974) 896; D10 (1974) 910.

[19] S. Deser and R.I. Nepomechie, Phys. Lett. B132 (1983) 321; Ann.Phys. 154 (1984) 396.

[20] A. Higuchi, Nucl.Phys. B282 (1987) 397.

[21] I. Bengtsson, J. Math. Phys. 36 (1995) 5806.

[22] I.L. Buchbinder, D.M. Gitman, V.A. Krykhtin and V.D. Pershin, Nucl. Phys. B584 (200) 615;

I.L. Buchbinder, D.M. Gitman and V.D. Pershin, Phys. Lett. B492 (2000) 161.

[23] Yu.M. Zinoviev, hep-th/0108192.

[24] I.L. Buchbinder, P.M. Lavrov and V.A. Krykhtin, Nucl. Phys. B762 (2007)344;

I.L. Buchbinder, V.A. Krykhtin and A.A. Reshetnyak, hep-th/0703049.

[25] S.V. Kuzmin and D.G.C. McKeon, Mod. Phys. Lett. A16 (2001) 747;

B. Kors and P. Nath, Phys. Lett. B586 (2004) 366; JHEP 0507 (2005) 069;

D.G.C. McKeon and T.J. Marshall, hep-th/0610034. 